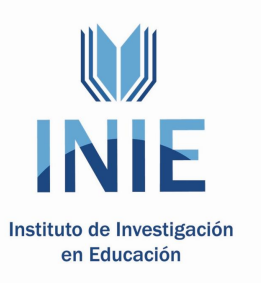

Actualidades Investigativas en Educación

Revista Electrónica publicada por el

Instituto de Investigación en Educación

Universidad de Costa Rica

ISSN 1409-4703

http://revista.inie.ucr.ac.cr

COSTA RICA

\title{
EL USO DE LAS NUEVAS TECNOLOGIAS DE LA INFORMACIÓN Y LA COMUNICACIÓN (NTICs) EN LA ENSEÑANZA DE LA FÍSICA MODERNA
}

THE USE OF NEW TECHNOLOGIES OF INFORMATION AND COMMUNICATION

TECHNOLOGIES (ICTS) IN THE TEACHING OF MODERN PHYSICS

\author{
Volumen 10, Número 1 \\ pp. 1-13
}

Este número se publicó el 30 de abril de 2010

Leda Roldán Santamaría

La revista está indexada en los directorios:

LATINDEX, REDALYC, IRESIE, CLASE, DIALNET, DOAJ, E-REVIST@S,

La revista está incluida en los sitios:

REDIE, RINACE, OEI, MAESTROTECA, PREAL, HUASCARAN, CLASCO

Los contenidos de este artículo están bajo una licencia Creative Commons 


\title{
EL USO DE LAS NUEVAS TECNOLOGIAS DE LA INFORMACIÓN Y LA COMUNICACIÓN (NTICs) EN LA ENSEÑANZA DE LA FÍSICA MODERNA \\ THE USE OF NEW TECHNOLOGIES OF INFORMATION AND COMMUNICATION TECHNOLOGIES (ICTS) IN THE TEACHING OF MODERN PHYSICS
}

\section{Leda María Roldán Santamaría ${ }^{1}$}

\begin{abstract}
Resumen: Este trabajo consiste en una reseña de una experiencia de aula donde se aprovecha el conocimiento en el campo de las Tecnologías de la Información y la Comunicación (TICs) para que los estudiantes del Colegio Científico Costarricense, con sede en San Pedro, San José de Costa Rica, logren investigar sobre temáticas de física moderna y comprender los principios básicos del tema de su interés. Las investigaciones en temáticas que presenta el programa de Física al concluir el undécimo año, involucraron a los estudiantes para que formaran parejas para realizar estos estudios sobre diversos temas. Indagaron en la WEB, capturaron imágenes que les permitieran reflejar los conceptos estudiados. Crearon afiches con los textos y las figuras que lograron relacionar, asimismo las presentaron a sus compañeros de colegio, respondiendo las preguntas que estos temas generaban en los espectadores.
\end{abstract}

Palabras claves: COLEGIOS CIENTÍFICOS, USO DE LAS TICS, ENSEÑANZA DE LA FÍSICA MODERNA, COSTA RICA

\begin{abstract}
This job to a review of a classroom where leverages knowledge in the field of ICTs that Scientific of Costa Rica High Scholl with headquarters in San Pedro, San José, Costa Rica, students achieve research on modern physics topics and understand the basics of the topic of your interest experience. Small research issues featuring the physics program at the end of 11th year involved students to form pairs of researchers on various topics. They searched the WEB on these issues, captured images allowing them to reflect the studied concepts. Created posters with texts and figures achieved relate and presented them to school, fellow answering the questions that these topics generated in others students.
\end{abstract}

KEY WORD: SCIENTIFIC HIGH SCHOLL, USE OF NTICES, TEACHING OF THE MODERN PHYSICS, COSTA RICA

\footnotetext{
${ }^{1}$ Maestría en Planificación Curricular, Licenciatuera en Física, ambos títulos de la Universidad de Costa Rica. Profesora Jubilada de la Escuela de Formación Docente de la Universidad de Costa Rica. Profesora de Física del Colegio Científico Costarricense, Sede San Pedro.
}

Dirección electrónica: leda.roldan@ucr.ac.cr

Artículo recibido: 2 de setiembre, 2009

Aprobado: $1^{\circ}$ de marzo, 2010 


\section{INTRODUCCIÓN}

Los Colegios Científicos Costarricenses (CCC) son instituciones de educación que conducen al bachillerato en enseñanza media, pero, al mismo tiempo, son consideradas instituciones parauniversitarias. Trabajan con dos niveles de enseñanza: décimo y undécimo año, con un solo grupo de cada nivel y un máximo de 30 estudiantes por aula. Eso permite que los docentes puedan trabajar con mayor comodidad cada uno de los programas del currículum de estas instituciones.

Estas nacen el 12 de abril de 1989 por decreto ejecutivo del Ministerio de Ciencia y Tecnología (MICYT) y el Ministerio de Educación Pública (MEP). Surgen como una novedosa alternativa para una educación de calidad, dando como resultado que sean los centros más exitosos dentro de las instituciones de secundaria públicas del MEP.

En el año 1989 surgieron solo dos instituciones de este tipo. Con el paso de los años, se fueron abriendo más en diferentes ubicaciones geográficas del país. Hoy, en total son nueve colegios científicos distribuidos en todo el territorio nacional, que ubicamos en el siguiente mapa 


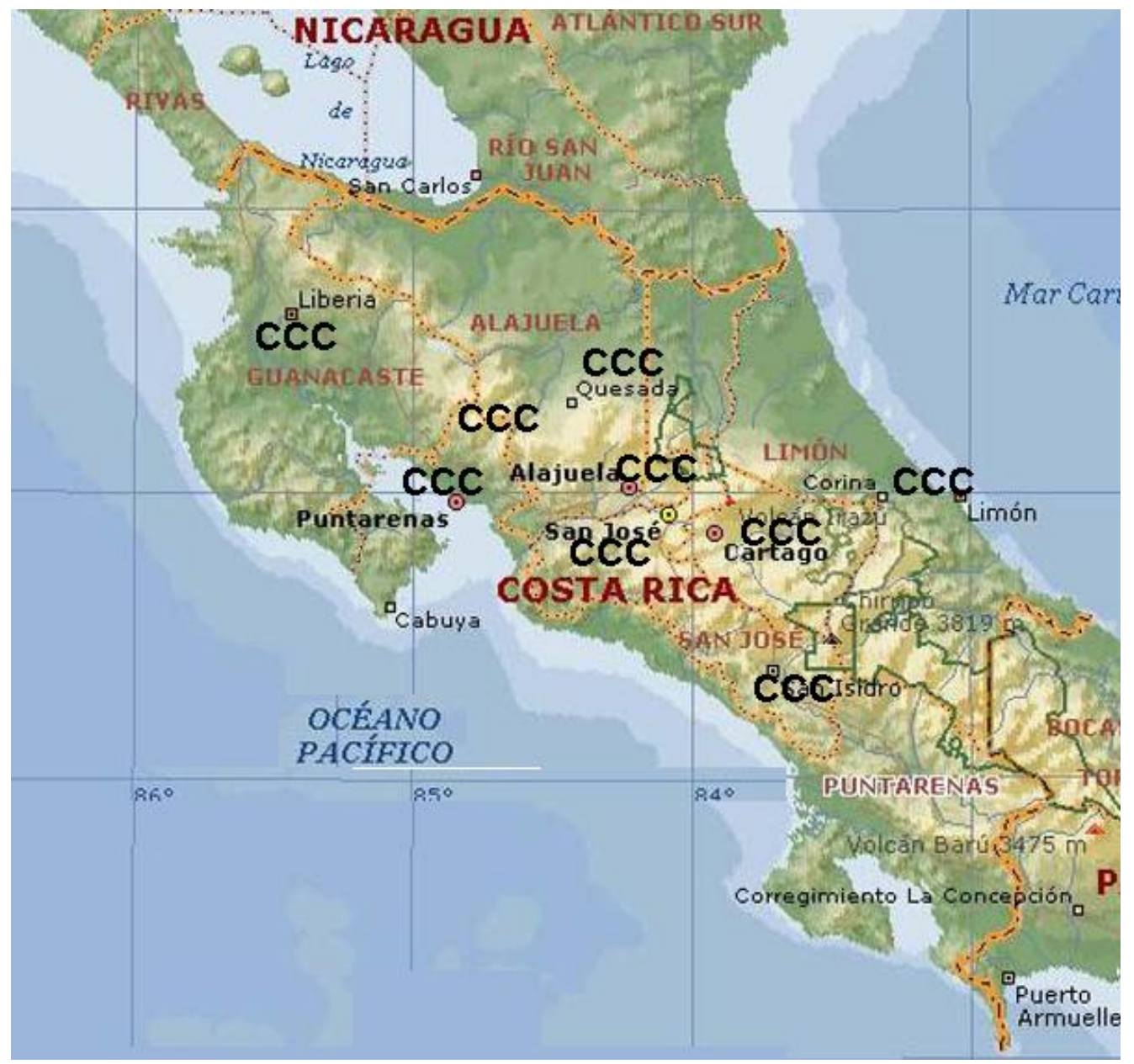

El objetivo general de los CCC es la formación integral de sus estudiantes, considerando los más altos valores costarricenses que conducen a la búsqueda permanente de la verdad, la justicia, la honestidad, la solidaridad, el compañerismo y un gran aprecio y cariño por la Patria y en el marco de un proceso educativo con énfasis en la adquisición de conocimientos sólidos y habilidades en los fundamentos de la matemática, la física, la química, la biología y la informática.

Sus estudiantes se destacan en concursos nacionales de Matemática, Biología y Física, así como en la participación de ferias Científicas, actividades en las cuales se presentan proyectos en el área de la ciencia y la tecnología, generados por los mismos estudiantes. También, estas instituciones ocupan los primeros lugares en la promoción de bachillerato, prueba a nivel nacional que aplica el MEP como control de calidad. 
Para ingresar al Sistema de Colegios Científicos Costarricenses, los estudiantes se deben inscribir en un proceso de selección para presentar el examen de admisión al sistema. Tendrán derecho a este examen aquellos estudiantes, cuyo rendimiento académico y de conducta, en los tres primeros años de secundaria, sea de muy bueno a excelente. Aquellos que se inscriben y cumplen con estas condiciones presentan un examen que comprende cuatro áreas: analogías, conocimientos científicos, conocimientos matemáticos y análisis de figuras.

De todos los estudiantes que presentan el examen, cada sede escoge las mejores 30 notas que ingresan $a^{\circ}$ año, en undécimo año no ingresan estudiantes nuevos.

Los estudiantes que permanecen en el sistema logran salir con excelentes conocimientos en el área científica y matemática, además de muchas habilidades en el campo de la investigación y la informática, como la capacidad de llevar bitácoras ordenadas con información confiable y aplicar conocimientos en el campo de la informática. Algunos estudiantes desertan por rendimiento académico o porque no logran adaptarse al modelo de estudios de los colegios, ya que, en estos casos, les resulta muy fuerte. Los que permanecen son estudiantes muy demandantes y les gusta enfrentarse a retos, de ahí que cada docente debe ingeniárselas para ofrecer una formación científica de calidad y con alta tecnología.

Ante la demanda de formación científica excelente, el uso de las tecnologías de la información y de la comunicación son de uso común en estos ambientes; para estos estudiantes navegar en internet en busca de información confiable y segura es cosa de todos los días.

\section{REFERENTE TEÓRICO}

\section{LAS TECNOLOGÍAS DE LA INFORMACIÓN Y LA COMUNICACIÓN}

Las tecnologías de la información y la comunicación (TICs) corresponden a un conjunto de servicios, redes, software y aparatos, cuyo objetivo es la mejora de la calidad de vida de las personas dentro de un entorno, y que se integran a un sistema de información interconectado y complementario. 
Las tecnologías de la información y la comunicación denominadas NTICs son métodos y recursos que se pueden utilizar igual que las TICs, pero que según el autor que se revise, pueden ser consideradas novedosas, ya que la tecnología avanza muy rápido y las NTICs utilizan otras herramientas que las TICs no hacen.

Las Tecnologías de la Información y la Comunicación (TICs) son un solo concepto en dos vertientes diferentes como principal premisa de estudio en las ciencias sociales, donde tales tecnologías afectan la forma de vivir de las sociedades. Su uso y abuso exhaustivo, para denotar modernidad, ha llevado a visiones totalmente erróneas del origen del término.

Las TIC se conciben como el universo de dos conjuntos representados por las tradicionales Tecnologías de la Comunicación (TC) -constituidas principalmente por la radio, la televisión y la telefonía convencional- y por las Tecnologías de la información (TI) caracterizadas por la digitalización de las tecnologías de registros de contenidos (informática, de las comunicaciones, telemática y de las interfaces).

Las TICs son herramientas teórico conceptuales, soportes y canales que procesan, almacenan, sintetizan, recuperan y presentan información de la forma más variada.

Los soportes han evolucionado en el transcurso del tiempo (telégrafo óptico, teléfono fijo, celulares, televisión) ahora, en esta era, podemos hablar de la computadora y de la Internet o red mundial. El uso de las TICs representa una variación notable en la sociedad y, a la larga, un cambio en la educación, en las relaciones interpersonales y en la forma de difundir y generar conocimientos.

\section{LAS TICs Y LAS NTICs}

También, es común comprender las TICs en relación con su magnitud (Internet o red mundial). Las TICs o NTICs tienen por objeto aportar claridad para conceptos aun más abstractos. El surgimiento de nuevos conocimientos, especialmente Internet, está transformando las posibilidades de acceso a la información en el mundo entero, cambiando nuestra manera de comunicarnos y también las rutinas diarias en los ámbitos de trabajo. 
La utilización de estas herramientas en la información y en la formación de nuevos conceptos ha fortalecido la enseñanza de la física. Los estudiantes de niveles superiores pueden tener acceso a información académica que en tiempo regular no estaría a su alcance. Uno de esos campos del conocimiento es la Física moderna, un campo muy actual en algunos aspectos, y que no siempre se encuentran en los libros de texto a nivel de secundaria.

\section{FÍSICA MODERNA}

Este campo de las ciencias físicas se ha establecido a inicios del siglo XX, cuando Planck investiga sobre el cuanto de energía. Dentro de este campo de estudio se han incluido conceptos que tienen que ver con el microcosmos, donde se consideran las nano partículas, cuerpos tan pequeños que han despertado el interés de las empresas que diseñan los chips miniaturizados. También, se considera el macrocosmos, donde los científicos se interesan por el estudio del Universo externo, es decir, el que rodea al Planeta Tierra.

Dentro de los campos de la Física Moderna, que estudian los estudiantes de secundaria, siguiendo el programa que propone el Ministerio de Educación Pública, se pueden mencionar:

$\checkmark$ Teoría especial de la relatividad

$\checkmark$ Teoría general de la relatividad

$\checkmark$ Experimento de Michelson y Morley

$\checkmark$ Ondas y fotones

$\checkmark$ Cuantización de la energía

$\checkmark$ Efecto fotoeléctrico

$\checkmark$ Comportamiento dual de la luz.

$\checkmark$ Ondas de Louis de Broglie.

$\checkmark$ Principio de incertidumbre de Heisenberg

La construcción de estos conocimientos en el campo de las NTICs y la física moderna, se aprovecharon para que los estudiantes del CCC de San Pedro lograrán diseñar afiches con los temas antes mencionados, siguiendo la metodología que se menciona a continuación. 


\section{METODOLOGÍA DE TRABAJO}

Tomando en cuenta el programa de estudio en el campo de la Física Moderna y los conocimientos previos de los estudiantes se propuso una experiencia cuya metodología se presenta en este apartado.

Como experiencia de aula, se les propuso a los estudiantes de último año de secundaria, del Colegio Científico Costarricense, sede San Pedro, que escogieran uno de los temas anteriores y que se unieran en parejas para hacer investigación con el uso de las NTICs.

Todos los grupos de dos estudiantes contaron con dos semanas para navegar en la Internet, para ubicar páginas con información confiable y redactar la

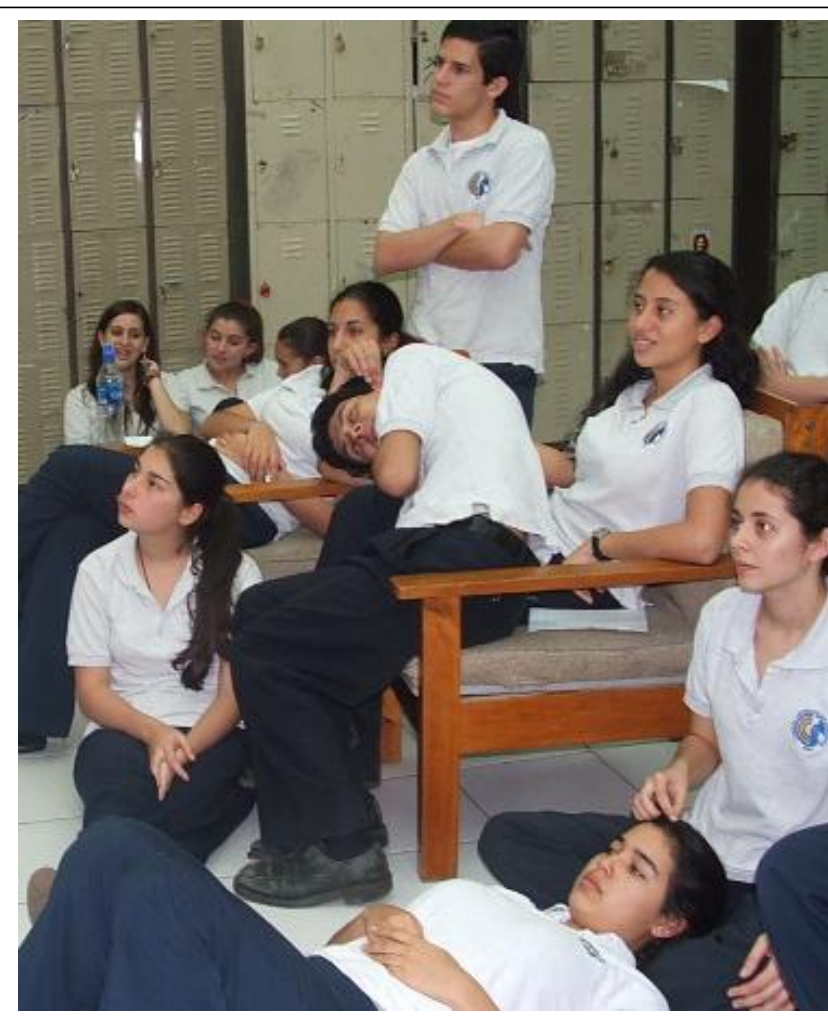

Grupo de estudiantes escuchando la exposición sobre el información que utilizarían para crear un afiche de dimensiones equivalentes a dos hojas carta.

Para identificar qué información tomar en cuenta, se entrevistaron con la productora de un programa que se ofrece en Radio de la Universidad de Costa Rica, cuyo título es ñ́Ciencia y Tecnología. Cosas de todos los díasò (http://cienteccr.blogspot.com). Este es un programa que presenta temáticas de ciencia y su duración es de cinco minutos. Esta persona les sugirió cómo lograr sintetizar la información y cómo seleccionarla. Además, les dio sugerencias de cómo ubicar el material en el diseño del afiche. Este debía ser atractivo, informativo y novedoso.

Una vez seleccionada la información, la clasificaron según el campo del conocimiento de la física que tratase: conceptos, aplicaciones modernas, científicos relacionados con el campo de interés, ventajas y desventajas, entre otros. 
Cuando todo el material estaba disponible se dieron a la tarea de diseñar el afiche con el programa r̃Publisherò de Microsoft, material que se encuentra disponible en las computadoras del laboratorio de cómputo del colegio. Durante las lecciones de física se usó el laboratorio para que pudieran utilizarlo por más tiempo. Los estudiantes discutieron cómo debían ubicar la información que ya tenían, cómo redactarla para que fuera de fácil comprensión para otros.

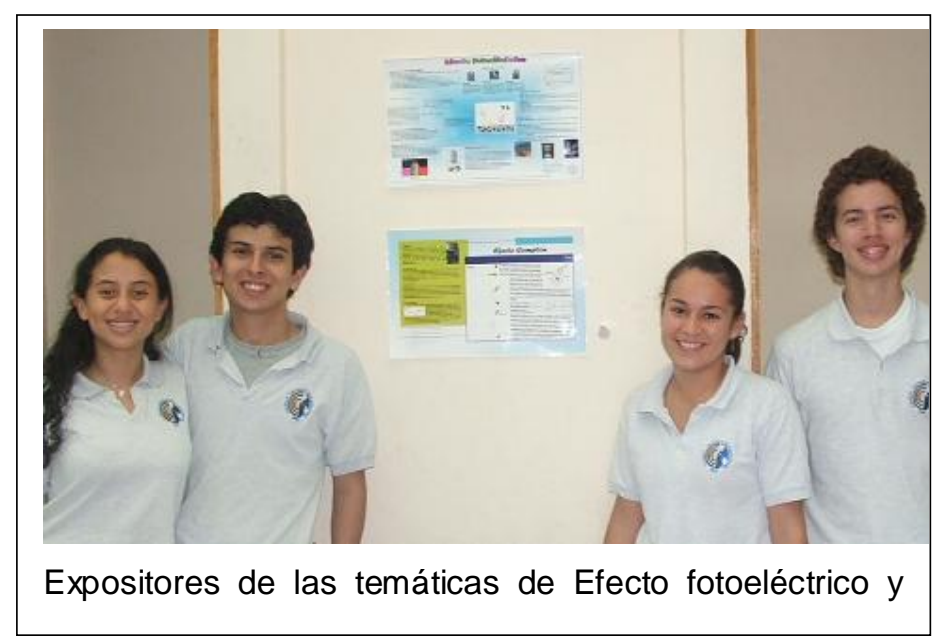

Concluido el diseño, e insertada la información, presentaron a la docente el material para su revisión. Aquí debe tomarse en cuanta que la docente, además de tener una amplia experiencia en el campo de la enseñanza de la Física, también cuenta con una Licenciatura en Física. Una vez hechas las correcciones sugeridas, los afiches se imprimieron y se emplasticaron para poderlos exhibir sin peligro de que se dañaran.

El día final del proyecto, cada pareja expuso su trabajo ante los demás compañeros y compañeras. Tanto de undécimo año como a los estudiantes de décimo año que deseaban saber sobre el tema.

Cada pareja expuso por veinte minutos su temática y demostraron un buen dominio de la misma, ya que los compañeros y compañeras formularon preguntas de un nivel de dificultad adecuada a la temática y las respuestas fueron muy interesantes y apropiadas para la dificultad.

\section{RESULTADOS Y DISCUSIÓN}

Los afiches fueron de alta calidad y los temas desarrollados por los estudiantes cumplieron con las expectativas. Algunos de estos afiches se pueden apreciar en los anexos. 
La exposición de los afiches se mantuvo por una semana, de manera que los estudiantes de décimo año lograron apreciar dicho trabajo e incluso hacerles preguntas a sus compañeros. Algunas de ellas versaban alrededor de cómo la física moderna afecta a nuestras vidas, la influencia de la física moderna en el campo de la astrofísica y hasta donde la teoría de la relatividad puede ser presentada a través de paradojas. Algunos estudiantes presentaron los afiches con figuras o formas con las

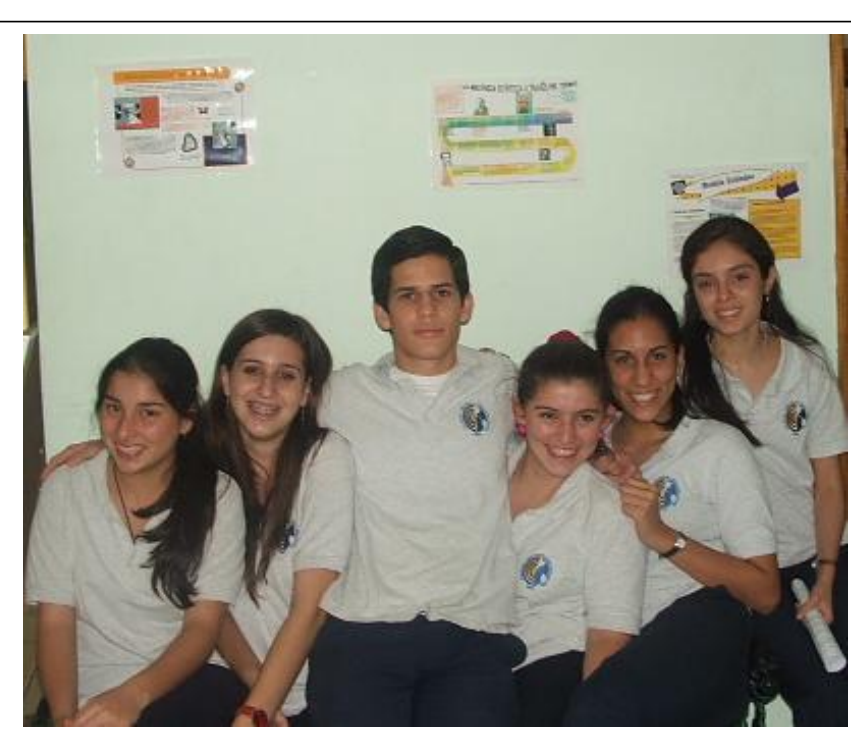

Expositoras y expositor de las temáticas sobre Principio de incertidumbre, Modelo estándar y línea del tiempo de la que querían presentar el significado del concepto, por ejemplo: el afiche sobre el principio de incertidumbre presenta una foto de Heisemberg al revés, lo presentaron así para dar a entender que las cosas se interpretan de acuerdo al nivel de referencia.

Las discusiones de los estudiantes sobre los cuestionamientos dejaron inquietudes en los estudiantes de décimo, de manera que ya se están organizando para las temáticas del próximo curso lectivo.

\section{CONCLUSIONES}

La idea de la utilización de las NTICs para la enseñanza de la física moderna apenas empieza, los estudiantes de décimo año quedaron muy interesados en las temáticas, algunos incluso empezaron a buscar nuevos temas usando las NTICs. Y si a eso se le suma que el proyecto del acelerador de partículas europeo ha estado en las noticias en las últimas semanas, estos estudiantes se han interesado mucho por la temática de partículas y el modelo estándar.

Además, los estudiantes de décimo año empezaron a recopilar información sobre los aspectos que se deben tener presentes para lograr un mayor provecho de las NTICs en la 
divulgación de conocimiento científico. Han conocido la aplicación en el campo de laboratorios virtuales y hasta donde estos son técnicas que permiten adquirir un conocimiento científico confiable. También se han interesado en utilizar esta red mundial como medio de ubicación de información confiable.

Por lo anterior, es un hecho que la idea del uso de las NTICs seguirá el próximo año, quizá no solo para física moderna sino para otros temas de interés de los estudiantes.

\section{AGRADECIMIENTOS}

Agradezco a los estudiantes de $11^{\circ}$ año 2008 del Colegio Científico Costarricense, sede San Pedro por el entusiasmo en el trabajo aquí publicado.

\section{REFERENCIAS}

Affronti, Mariana. (s.f.). Aprender con las NTICs. Recuperado en julio 2008 de http://maffronti.blogspot.com/

López, Alexander. (2001). ¿Son un peligro las NTIC? Problemas socioeconómicos, políticos, culturales y éticos. Contexto Educativo, Año III (19). Recuperado en julio 2008, de http://contexto-educativo.com.ar/2001/5/nota-10.htm

Mainieri, Aida, Roldán, Leda y Minero, Eduardo. (1999). Colegios Científicos Costarricenses. Una experiencia educativa. En Alicia Gurdián (comp.), Política Social y Educación en Costa Rica (pp. 365-419). San José, C.R.: UNICEF

\section{ANEXOS}

Material generado por el grupo de estudiantes de $11^{\circ}$ año del Colegio Científico Costarricense, Sede San Pedro. 


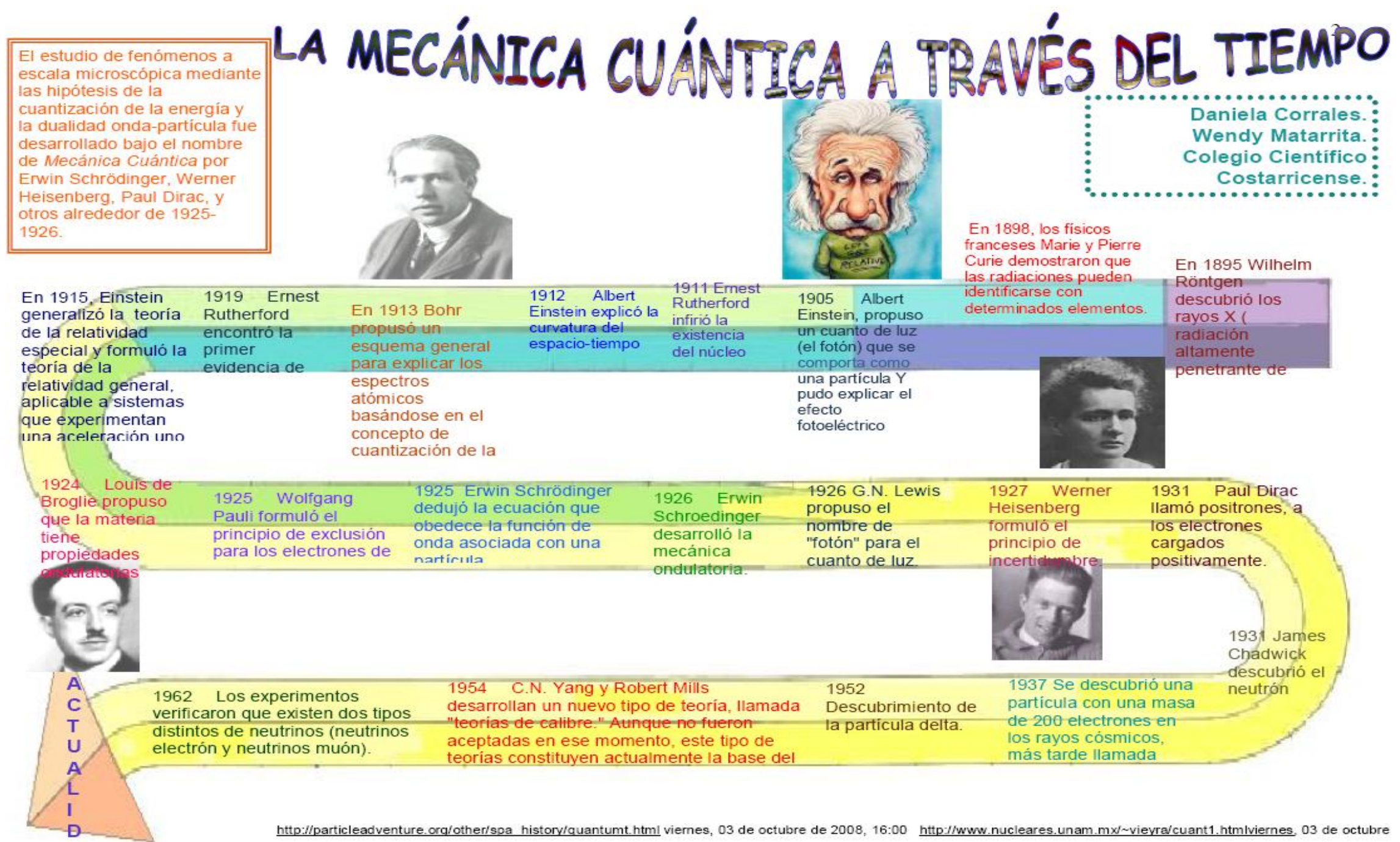




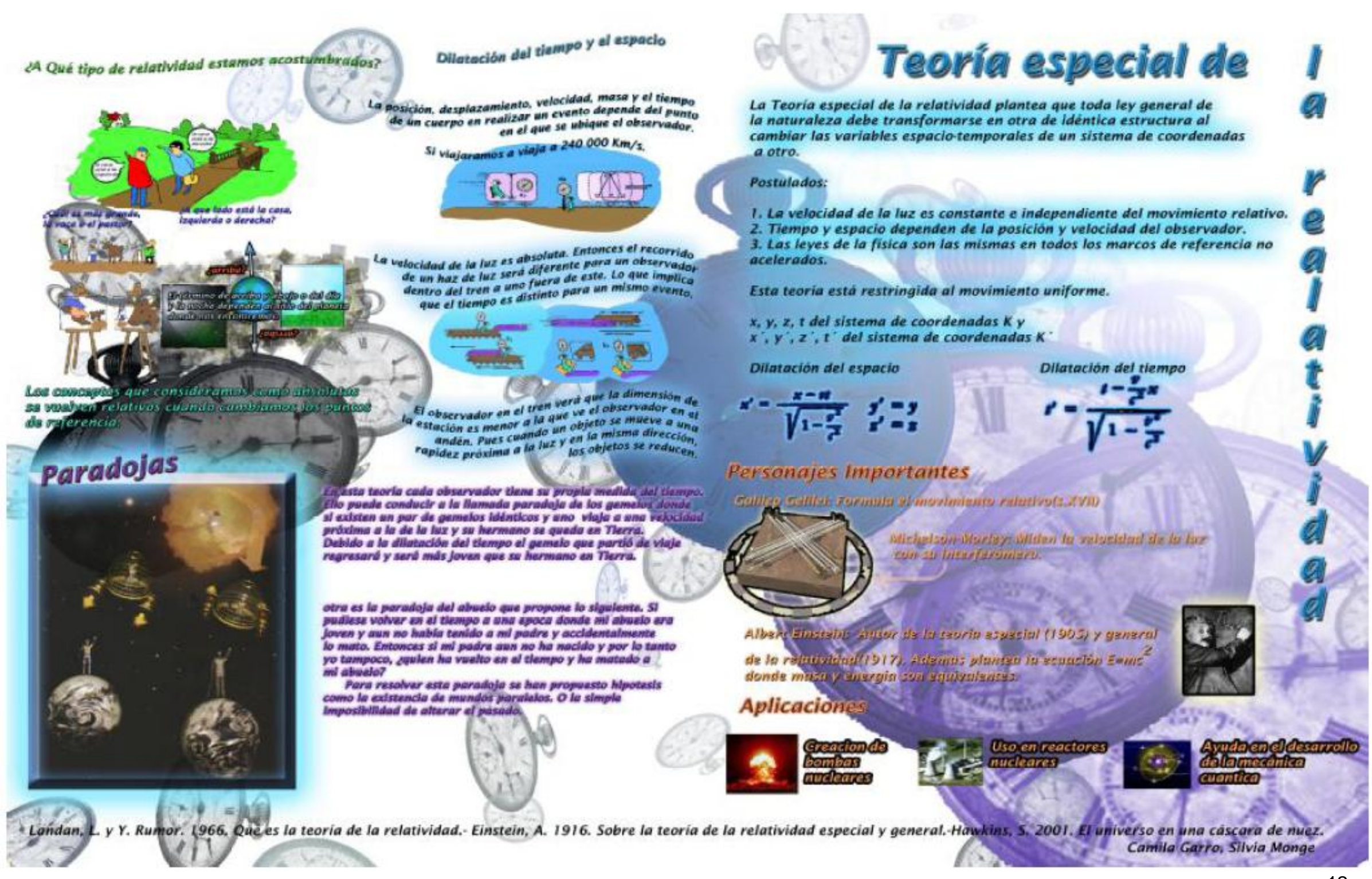




\section{PRINCIPIO DE INCERTIDUIMBRE}

En la mecánica cuántica, conocer dos propiedades que permitan identificar con exactitud a la partícula es imposible al mismo tiempo. ¿Jugará Dios a los aados?

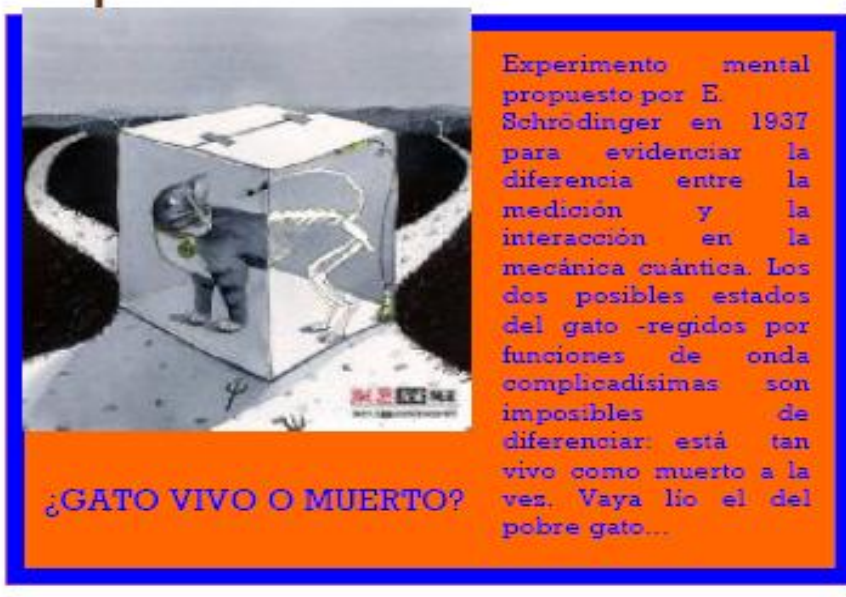

Se puede obtener una formulación apropiada del principio de incertidumbre en la frecuencia $\Delta f$ de una onda en un periodo finito de tiempo $\Delta t$ en el cual se observa la onda y se mide su frecuencia. En este caso la onda es periódica de extensión infinita en el espacio. Una onda restringida a una región finita del espacio no puede caracterizarse con una frecuencia o una longitud bien definida. En forma semejante, la relación se puede aplicar entre la incertidumbre del ímpetu de la partícula $\mathrm{v}$ su loncitud de onda.

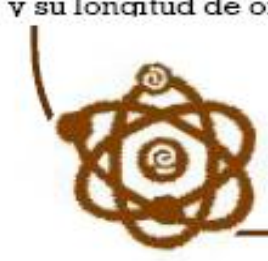

En la actualidad, el principio de incertidumbre es aplicado en múltiples áreas. Es importante para el cálculo de tablas de interés y amortización. En el LHC del CERN, ubicado en Francia-Suiza, la incertidumbre toma un valor importante para el cálculo de la posición de las partículas en los choques provocados.

Suponga que usted llama a un teléfono fijo, en este caso usted sabría con certeza a que lugar está llamando. Sin embargo, no tiene una idea de quién le va a contestar. Por otra parte, si usted llama a un celular, estará seguro de quién le contestará, pero no podrá precisar el lugar donde esta la persona que contesta.

-Si usted desea saber la posición de un electrón tiene que Lura averiguarlo. La sola acción de un fotón

sobre el electrón le imprimiría

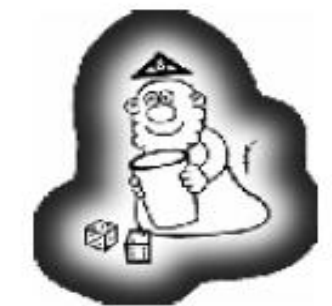

Einstein refutó ampliamente el principio porque según el "Dios no juega a los dados"
$\Delta E \Delta t \geq h$
$\Delta \mathrm{px} \Delta \mathrm{x} \geq \mathrm{h}$

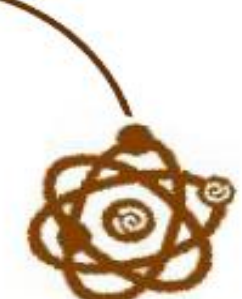

। a a. 